\title{
DETERMINANTAL EXPRESSIONS AND RECURRENCE RELATIONS FOR FUBINI AND EULERIAN POLYNOMIALS
}

\author{
FENG QI
}

Institute of Mathematics, Henan Polytechnic University, Jiaozuo 454010, Henan, China; College of Mathematics, Inner Mongolia University for Nationalities, Tongliao 028043, China; School of Mathematical Sciences, Tianjin

Polytechnic University, Tianjin 300387, China

E-mail: qifeng618@gmail.com, qifeng618@hotmail.com,qifeng618@qq.com

URL: http: // qifeng618. wordpress. com

\begin{abstract}
In the paper, by virtue of an explicit formula for higher order derivatives of the ratio between two differentiable functions in terms of the Hessenberg determinant, the author presents determinantal expressions and recurrence relations for generalized Fubini polynomials. Hereafter, by virtue of relations between Eulerian polynomials and the (generalized) Fubini numbers and polynomials, the author derives a determinantal expression for Eulerian polynomials and several recurrence relations for the Fubini and Eulerian polynomials.

AMS subject classifications (2010): Primary 11B68; Secondary 11B37, 11B83, 26A24, 26C05, $33 \mathrm{~B} 10$.

Keywords: generalized Fubini polynomial; Eulerian polynomial; determinantal expression; recurrence relation; Hessenberg determinant.
\end{abstract}

\section{INTRODUCTION}

For $n \geq 0$, let

$$
F_{n}=\sum_{k=0}^{n} k ! S(n, k)
$$

where $S(n, k)$, which can be generated by the exponential function

$$
\frac{\left(e^{x}-1\right)^{k}}{k !}=\sum_{n=k}^{\infty} S(n, k) \frac{x^{n}}{n !}
$$

and can be computed by the explicit formula

$$
S(n, k)=\frac{1}{k !} \sum_{\ell=1}^{k}(-1)^{k-\ell}\left(\begin{array}{l}
k \\
\ell
\end{array}\right) \ell^{n},
$$

denotes the Stirling numbers of the second kind [16. One calls these numbers $F_{n}$ the Fubini numbers [7, ordered Bell numbers [10], or geometric numbers [36]. A Fubini number $F_{n}$ has been interpreted in [10, 36] combinatorially: it counts all the possible set partitions of an $n$ element set such that the order of the blocks matters. In [10], the Fubini numbers $F_{n}$ were connected

Please cite this article as "Feng Qi, Determinantal expressions and recurrence relations for Fubini and Eulerian polynomials, Journal of Interdisciplinary Mathematics 22 (2019), no. 3, 317-335; Available online at https://doi. org/10.1080/09720502.2019.1624063"

This paper was typeset using $\mathcal{A M}_{\mathcal{S}}$-LATEX. 
with preference arrangements and the recursion for $F_{n}$ was derived. In [10, 11, the exponential generating function

$$
G(t)=\frac{1}{2-e^{t}}=\sum_{n=0}^{\infty} F_{n} \frac{t^{n}}{n !}
$$

and an asymptotic estimate for $F_{n}$ were established.

In [36], the Fubini polynomials $F_{n}(y)$ were defined by

$$
F_{n}(y)=\sum_{k=0}^{n} S(n, k) k ! y^{k} .
$$

These polynomials can be generated [36, pp. 735-736] by

$$
G(t, y)=\frac{1}{1-y\left(e^{t}-1\right)}=\sum_{n=0}^{\infty} F_{n}(y) \frac{t^{n}}{n !} .
$$

It is clear that $F_{n}(1)=F_{n}$. Due to the relation

$$
\left(y \frac{\mathrm{d}}{\mathrm{d} y}\right)^{m} \frac{1}{1-y}=\sum_{k=0}^{\infty} k^{m} y^{k}=\frac{1}{1-y} F_{m}\left(\frac{y}{1-y}\right), \quad|y|<1
$$

in [2, one also calls $F_{n}(y)$ the geometric polynomials.

In [12, Section 2], the Fubini polynomials $F_{n}(x, y)$ of two variables $x, y$ are defined by means of the generating function

$$
G(t, y, x)=\frac{e^{x t}}{1-y\left(e^{t}-1\right)}=\sum_{n=0}^{\infty} F_{n}(x, y) \frac{t^{n}}{n !} .
$$

It is apparent that $F_{n}(0, y)=F_{n}(y)$.

In [13], the bivariate Fubini polynomials $F_{n}^{(r)}(x, y)$ of order $r$ for $r \in \mathbb{N}$, generated by

$$
G(t, y, x, r)=\frac{e^{x t}}{\left[1-y\left(e^{t}-1\right)\right]^{r}}=\sum_{n=0}^{\infty} F_{n}^{(r)}(x, y) \frac{t^{n}}{n !},
$$

were studied. It is obvious that $F_{n}^{(1)}(x, y)=F_{n}(x, y)$.

The generating functions of $F_{n}, F_{n}(y), F_{n}(x, y)$, and $F_{n}^{(r)}(x, y)$ remind us to consider the generating function

$$
G(t, y, x, q, z)=\frac{e^{x t}}{\left[z-y\left(e^{t}-1\right)\right]^{q}}=\sum_{n=0}^{\infty} F_{n}(x, y, z, q) \frac{t^{n}}{n !}, \quad x, q \in \mathbb{R}
$$

and generalized Fubini polynomials $F_{n}(x, y, z, q)$ of four variables $x, y, z, q$. It is clear that, since

$$
G(t, y, x, q, z)=\frac{1}{z^{q}} G\left(t, \frac{y}{z}, x, r\right)
$$

we have

$$
F_{n}(x, y, z, r)=\frac{F_{n}^{(r)}(x, y / z)}{z^{r}} .
$$

In this paper, we will establish some properties of generalized Fubini polynomials $F_{n}(x, y, z, q)$ and, consequently, derive some known results. 


\section{A NeW Determiantal expression of the Fubini polynomials}

At first, we simply establish a new determinantal expressions of the Fubini polynomials and numbers $F_{n}(y)$ and $F_{n}$ respectively.

Theorem 2.1. The Fubini polynomials $F_{n}(y)$ for $n \geq 0$ can be represented determinantally by

$$
F_{n}(y)=\left|\begin{array}{cccccccc}
1 & -1 & 0 & 0 & \cdots & 0 & 0 & 0 \\
0 & y & -1 & 0 & \cdots & 0 & 0 & 0 \\
0 & y & \left(\begin{array}{l}
2 \\
1
\end{array}\right) y & -1 & \cdots & 0 & 0 & 0 \\
0 & y & \left(\begin{array}{l}
3 \\
1
\end{array}\right) y & \left(\begin{array}{l}
3 \\
2
\end{array}\right) y & \cdots & 0 & 0 & 0 \\
\cdots & \cdots & \cdots & \cdots & \ddots & \cdots & \cdots & \cdots \\
0 & y & \left(\begin{array}{c}
n-2 \\
1
\end{array}\right) y & \left(\begin{array}{c}
n-2 \\
2
\end{array}\right) y & \cdots & \left(\begin{array}{c}
n-2 \\
n-3
\end{array}\right) y & -1 & 0 \\
0 & y & \left(\begin{array}{c}
n-1 \\
1
\end{array}\right) y & \left(\begin{array}{c}
n-1 \\
2
\end{array}\right) y & \cdots & \left(\begin{array}{c}
n-1 \\
n-3
\end{array}\right) y & \left(\begin{array}{c}
n-1 \\
n-2
\end{array}\right) y & -1 \\
0 & y & \left(\begin{array}{c}
n \\
1
\end{array}\right) y & \left(\begin{array}{c}
n \\
2
\end{array}\right) y & \cdots & \left(\begin{array}{c}
n \\
n-3
\end{array}\right) y & \left(\begin{array}{c}
n \\
n-2
\end{array}\right) y & \left(\begin{array}{c}
n-1 \\
n-1
\end{array}\right) y
\end{array}\right| .
$$

Consequently, the Fubini numbers $F_{n}$ can be represented determinantally by

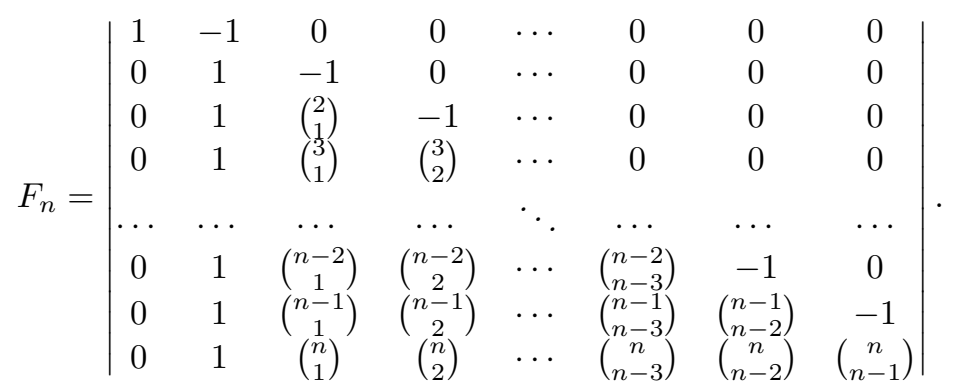

Proof. Let $u(t)$ and $v(t) \neq 0$ be differentiable functions, let $U_{(n+1) \times 1}(t)$ be an $(n+1) \times 1$ matrix whose elements $u_{k, 1}(t)=u^{(k-1)}(t)$ for $1 \leq k \leq n+1$, let $V_{(n+1) \times n}(t)$ be an $(n+1) \times n$ matrix whose elements

$$
v_{i, j}(t)= \begin{cases}\left(\begin{array}{l}
i-1 \\
j-1
\end{array}\right) v^{(i-j)}(t), & i-j \geq 0 ; \\
0, & i-j<0\end{cases}
$$

for $1 \leq i \leq n+1$ and $1 \leq j \leq n$, and let $\left|W_{(n+1) \times(n+1)}(t)\right|$ denote the lower Hessenberg determinant of the $(n+1) \times(n+1)$ lower Hessenberg matrix

$$
W_{(n+1) \times(n+1)}(t)=\left[U_{(n+1) \times 1}(t) \quad V_{(n+1) \times n}(t)\right] .
$$

Then the $n$th derivative of the ratio $\frac{u(t)}{v(t)}$ can be computed by

$$
\frac{\mathrm{d}^{n}}{\mathrm{~d} x^{n}}\left[\frac{u(t)}{v(t)}\right]=(-1)^{n} \frac{\left|W_{(n+1) \times(n+1)}(t)\right|}{v^{n+1}(t)} .
$$

See [1, p. 40, Exercise 5)], the papers [14, 21] and closely related references. Taking $u(t)=1$ and $v(t)=1-y\left(e^{t}-1\right)$ in 2.2 yields

$$
\frac{\mathrm{d}^{n}}{\mathrm{~d} t^{n}} G(t, y)=(-1)^{n} G^{n+1}(t, y)
$$




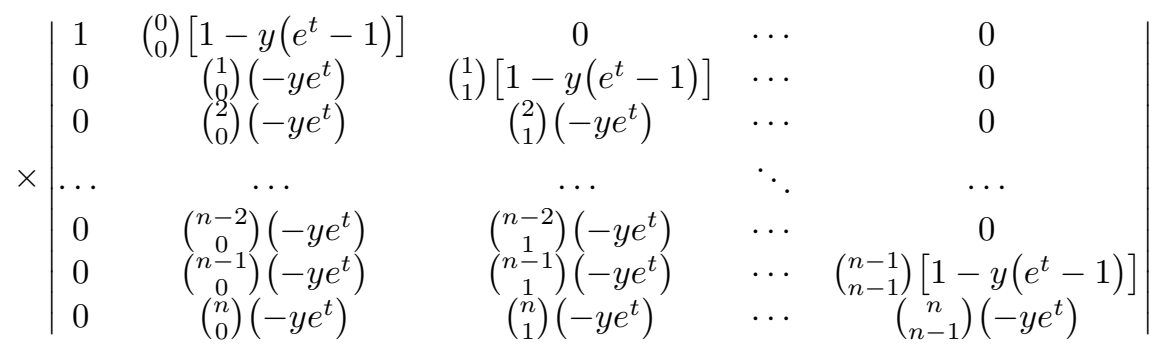

$$
\begin{aligned}
& \rightarrow(-1)^{n}\left|\begin{array}{ccccc}
1 & \left(\begin{array}{c}
0 \\
0 \\
0
\end{array}\right) & 0 & \cdots & 0 \\
0 & \left(\begin{array}{c}
1 \\
0
\end{array}\right)(-y) & \left(\begin{array}{l}
1 \\
1
\end{array}\right) & \cdots & 0 \\
0 & \left(\begin{array}{l}
2 \\
0
\end{array}\right)(-y) & \left(\begin{array}{l}
2 \\
1
\end{array}\right)(-y) & \cdots & 0 \\
\cdots & \cdots & \cdots & \ddots & \cdots \\
0 & \left(\begin{array}{c}
n-2 \\
0
\end{array}\right)(-y) & \left(\begin{array}{c}
n-2 \\
1
\end{array}\right)(-y) & \cdots & 0 \\
0 & \left(\begin{array}{c}
n-1 \\
0
\end{array}\right)(-y) & \left(\begin{array}{c}
n-1 \\
1
\end{array}\right)(-y) & \cdots & \left(\begin{array}{c}
n-1 \\
n-1
\end{array}\right) \\
0 & \left(\begin{array}{c}
n \\
0
\end{array}\right)(-y) & \left(\begin{array}{l}
n \\
1
\end{array}\right)(-y) & \cdots & \left(\begin{array}{c}
n \\
n-1
\end{array}\right)(-y)
\end{array}\right|, \quad t \rightarrow 0 \\
& =\left|\begin{array}{ccccc}
1 & -1 & 0 & \cdots & 0 \\
0 & \left(\begin{array}{c}
1 \\
0
\end{array}\right) y & -1 & \cdots & 0 \\
0 & \left(\begin{array}{c}
2 \\
0
\end{array}\right) y & \left(\begin{array}{c}
2 \\
1
\end{array}\right) y & \cdots & 0 \\
\cdots & \cdots & \cdots & \ddots & \cdots \\
0 & \left(\begin{array}{c}
n-2 \\
0
\end{array}\right) y & \left(\begin{array}{c}
n-2 \\
0
\end{array}\right) y & \cdots & 0 \\
0 & \left(\begin{array}{c}
n-1 \\
0
\end{array}\right) y & \left(\begin{array}{c}
n-1 \\
1
\end{array}\right) y & \cdots & -1 \\
0 & \left(\begin{array}{c}
n \\
n \\
0
\end{array}\right) y & \left(\begin{array}{c}
n \\
1
\end{array}\right) y & \cdots & \left(\begin{array}{c}
n \\
n-1
\end{array}\right) y
\end{array}\right| .
\end{aligned}
$$

The determinantal expression (2.1) is thus proved. The proof of Theorem 2.1 is complete.

\section{Two RECURREnCE RELATions of the Fubini polynomials}

Basing on Theorem 2.1 and the binomial inversion theorem, we now derive two recurrence relations of the Fubini polynomials $F_{n}(y)$.

Theorem 3.1. The Fubini polynomials $F_{n}(y)$ satisfy

$$
F_{n}(y)=y \sum_{r=0}^{n-1}\left(\begin{array}{l}
n \\
r
\end{array}\right) F_{r}(y), \quad n \in \mathbb{N}
$$

and

$$
F_{n}(y)=(-1)^{n+1}(1+y)\left[\sum_{k=0}^{n-1}(-1)^{k}\left(\begin{array}{l}
n \\
k
\end{array}\right) F_{k}(y)-\frac{1}{y}\right], \quad n \geq 0 .
$$


Proof. Let $M_{0}=1$ and

$$
M_{n}=\left|\begin{array}{cccccc}
m_{1,1} & m_{1,2} & 0 & \ldots & 0 & 0 \\
m_{2,1} & m_{2,2} & m_{2,3} & \ldots & 0 & 0 \\
m_{3,1} & m_{3,2} & m_{3,3} & \ldots & 0 & 0 \\
\vdots & \vdots & \vdots & \vdots & \vdots & \vdots \\
m_{n-2,1} & m_{n-2,2} & m_{n-2,3} & \ldots & m_{n-2, n-1} & 0 \\
m_{n-1,1} & m_{n-1,2} & m_{n-1,3} & \ldots & m_{n-1, n-1} & m_{n-1, n} \\
m_{n, 1} & m_{n, 2} & m_{n, 3} & \ldots & m_{n, n-1} & m_{n, n}
\end{array}\right|
$$

for $n \in \mathbb{N}$. Then the main theorem on page 222 in [5] reads that the sequence $M_{n}$ for $n \geq 0$ satisfies $M_{1}=m_{1,1}$ and

$$
M_{n}=m_{n, n} M_{n-1}+\sum_{r=1}^{n-1}(-1)^{n-r} m_{n, r}\left(\prod_{j=r}^{n-1} m_{j, j+1}\right) M_{r-1}, \quad n \geq 2 .
$$

Applying 3.3 to the determinantal expression 2.1) and simplifying yield the recurrence relation (3.1).

It is well known [6, pp. 143-144] that the binomial inversion theorem reads that

$$
s_{n}=\sum_{k=0}^{n}\left(\begin{array}{l}
n \\
k
\end{array}\right) S_{k} \quad \text { if and only if } \quad S_{n}=\sum_{k=0}^{n}(-1)^{n-k}\left(\begin{array}{l}
n \\
k
\end{array}\right) s_{k}
$$

for $n \geq 0$, where $s_{n}$ and $S_{n}$ are sequences of complex numbers. The relation (3.1) can be rearranged as

$$
\left(1+\frac{1}{y}\right) F_{n+1}(y)=\sum_{r=0}^{n+1}\left(\begin{array}{c}
n+1 \\
r
\end{array}\right) F_{r}(y), \quad n \geq 0 .
$$

Therefore, we obtain

$$
F_{n}(y)=\sum_{k=0}^{n}(-1)^{n-k}\left(\begin{array}{l}
n \\
k
\end{array}\right)\left(1+\frac{1}{y}\right) F_{k}(y)-\frac{(-1)^{n}}{y}, \quad n \geq 0
$$

which can be rewritten as 3.2 . The proof of Theorem 3.1 is complete.

\section{A DEterminantal eXPRESSiOn FOR Generalized Fubini POLYNOMials}

In this section, We establish a determinantal expression for generalized Fubini polynomials $F_{n}(x, y, z, q)$ of four variables $x, y, z, q$.

Theorem 4.1. Generalized Fubini polynomials $F_{n}(x, y, z, q)$ can be determinantally expressed as

$$
\begin{aligned}
& F_{n}(x, y, z, q)=\frac{(-1)^{n}}{z^{q(n+1)}} \\
& \times\left|\begin{array}{ccccc}
1 & \left(\begin{array}{l}
0 \\
0
\end{array}\right) z^{q} & 0 & \cdots & 0 \\
x & \left(\begin{array}{l}
1 \\
0
\end{array}\right) m_{1}(z, y, q) & \left(\begin{array}{l}
1 \\
1
\end{array}\right) z^{q} & \cdots & 0 \\
x^{2} & \left(\begin{array}{l}
2 \\
0
\end{array}\right) m_{2}(z, y, q) & \left(\begin{array}{l}
2 \\
1
\end{array}\right) m_{1}(z, y, q) & \ldots & 0 \\
\ldots & \ldots & \ldots & \ddots & \ldots \\
x^{n-2} & \left(\begin{array}{c}
n-2 \\
0
\end{array}\right) m_{n-2}(z, y, q) & \left(\begin{array}{c}
n-2 \\
1
\end{array}\right) m_{n-3}(z, y, q) & \ldots & 0 \\
x^{n-1} & \left(\begin{array}{c}
n-1 \\
0
\end{array}\right) m_{n-1}(z, y, q) & \left(\begin{array}{c}
n-1 \\
1
\end{array}\right) m_{n-2}(z, y, q) & \ldots & \left(\begin{array}{c}
n-1 \\
n-1
\end{array}\right) z^{q} \\
x^{n} & \left(\begin{array}{c}
n \\
0
\end{array}\right) m_{n}(z, y, q) & \left(\begin{array}{c}
n \\
1
\end{array}\right) m_{n-1}(z, y, q) & \cdots & \left(\begin{array}{c}
n \\
n-1
\end{array}\right) m_{1}(z, y, q)
\end{array}\right|
\end{aligned}
$$


and satisfy the recurrence relation

$$
F_{n}(x, y, z, q)=\frac{2}{z^{q}}\left[x^{n}-\sum_{r=0}^{n-1}\left(\begin{array}{l}
n \\
r
\end{array}\right) m_{n-r}(z, y, q) F_{r}(x, y, z, q)\right],
$$

where

$$
m_{k}(z, y, q)=\sum_{\ell=0}^{k}(-1)^{\ell} S(k, \ell)\langle q\rangle_{\ell} z^{q-\ell} y^{\ell}, \quad k \geq 0 .
$$

Proof. In 6, p. 134, Theorem A] and [6, p. 139, Theorem C], the Bell polynomials of the second kind $\mathrm{B}_{n, k}\left(x_{1}, x_{2}, \ldots, x_{n-k+1}\right)$ for $n \geq k \geq 0$ are defined by

$$
\mathrm{B}_{n, k}\left(x_{1}, x_{2}, \ldots, x_{n-k+1}\right)=\sum_{\substack{1 \leq i \leq n-k+1 \\ \ell_{i} \in\{0\} \cup \mathbb{N} \\ \sum_{i=k+1}^{n-k+1} i \ell_{i}=n \\ \sum_{i=1}^{n-k+1} \ell_{i}=k}} \frac{n !}{\prod_{i=1}^{n-k+1} \ell_{i} !} \prod_{i=1}^{n-k+1}\left(\frac{x_{i}}{i !}\right)^{\ell_{i}} .
$$

The Faà di Bruno formula can be described in terms of the Bell polynomials of the second kind $\mathrm{B}_{n, k}\left(x_{1}, x_{2}, \ldots, x_{n-k+1}\right)$ by

$$
\frac{\mathrm{d}^{n}}{\mathrm{~d} t^{n}} f \circ h(t)=\sum_{k=0}^{n} f^{(k)}(h(t)) \mathrm{B}_{n, k}\left(h^{\prime}(t), h^{\prime \prime}(t), \ldots, h^{(n-k+1)}(t)\right) .
$$

In [6, p. 135], it is given that

$$
\mathrm{B}_{n, k}\left(a b x_{1}, a b^{2} x_{2}, \ldots, a b^{n-k+1} x_{n-k+1}\right)=a^{k} b^{n} \mathrm{~B}_{n, k}\left(x_{1}, x_{2}, \ldots, x_{n-k+1}\right)
$$

and

$$
\mathrm{B}_{n, k}(1,1, \ldots, 1)=S(n, k)
$$

where $a$ and $b$ are complex numbers.

By the Faà di Bruno formula (4.3) and the identities (4.4) and 4.5), we obtain

$$
\begin{aligned}
\frac{\mathrm{d}^{k}}{\mathrm{~d} t^{k}}\left[z-y\left(e^{t}-1\right)\right]^{q} & =\sum_{\ell=0}^{k} \frac{\mathrm{d}^{\ell} u^{q}}{\mathrm{~d} u^{\ell}} \mathrm{B}_{k, \ell}\left(-y e^{t},-y e^{t}, \ldots,-y e^{t}\right) \\
& =\sum_{\ell=0}^{k}\langle q\rangle_{\ell} u^{q-\ell}\left(-y e^{t}\right)^{\ell} \mathrm{B}_{k, \ell}(1,1, \ldots, 1) \\
& =\sum_{\ell=0}^{k}\langle q\rangle_{\ell}\left[z-y\left(e^{t}-1\right)\right]^{q-\ell}\left(-y e^{t}\right)^{\ell} S(k, \ell) \\
& \rightarrow \sum_{\ell=0}^{k}(-1)^{\ell} S(k, \ell)\langle q\rangle_{\ell} z^{q-\ell} y^{\ell}
\end{aligned}
$$

as $t \rightarrow 0$, where $u=u(t)=z-y\left(e^{t}-1\right)$ and

$$
\langle q\rangle_{n}=\prod_{k=0}^{n-1}(q-k)= \begin{cases}q(q-1) \cdots(q-n+1), & n \geq 1 \\ 1, & n=0\end{cases}
$$

is the falling factorial of the number $q$. 
In the formula 2.2 , taking $u(t)=e^{x t}$ and $v(t)=\left[z-y\left(e^{t}-1\right)\right]^{q}$ yields

$$
\begin{aligned}
& \frac{\mathrm{d}^{n}}{\mathrm{~d} t^{n}} G(t, y, x, q, z)=\frac{(-1)^{n}}{\left[z-y\left(e^{t}-1\right)\right]^{q(n+1)}} \\
& \times\left|\begin{array}{ccccc}
e^{x t} & \left(\begin{array}{l}
0 \\
0
\end{array}\right)\left(z+y-y e^{t}\right)^{q} & 0 & \ldots & 0 \\
x e^{x t} & \left(\begin{array}{l}
1 \\
0
\end{array}\right)\left[\left(z+y-y e^{t}\right)^{q}\right]^{\prime} & \left(\begin{array}{l}
1 \\
1
\end{array}\right)\left(z+y-y e^{t}\right)^{q} & \ldots & 0 \\
x^{2} e^{x t} & \left(\begin{array}{l}
2 \\
0
\end{array}\right)\left[\left(z+y-y e^{t}\right)^{q}\right]^{\prime \prime} & \left(\begin{array}{l}
2 \\
1
\end{array}\right)\left[\left(z+y-y e^{t}\right)^{q}\right]^{\prime} & \ldots & 0 \\
\cdots & \ldots & \ldots & \ddots & \ldots \\
x^{n-2} e^{x t} & \left(\begin{array}{c}
n-2 \\
0
\end{array}\right)\left[\left(z+y-y e^{t}\right)^{q}\right]^{(n-2)} & \left(\begin{array}{c}
n-2 \\
1
\end{array}\right)\left[\left(z+y-y e^{t}\right)^{q}\right]^{(n-3)} & \ldots & 0 \\
x^{n-1} e^{x t} & \left(\begin{array}{c}
n-1 \\
0
\end{array}\right)\left[\left(z+y-y e^{t}\right)^{q}\right]^{(n-1)} & \left(\begin{array}{c}
n-1 \\
1
\end{array}\right)\left[\left(z+y-y e^{t}\right)^{q}\right]^{(n-2)} & \ldots & \left(\begin{array}{c}
n-1 \\
n-1
\end{array}\right)\left(z+y-y e^{t}\right)^{q} \\
x^{n} e^{x t} & \left(\begin{array}{c}
n \\
0
\end{array}\right)\left[\left(z+y-y e^{t}\right)^{q}\right]^{(n)} & \left(\begin{array}{c}
n \\
1
\end{array}\right)\left[\left(z+y-y e^{t}\right)^{q}\right]^{(n-1)} & \ldots & \left(\begin{array}{c}
n \\
n-1
\end{array}\right)\left[\left(z+y-y e^{t}\right)^{q}\right]^{\prime}
\end{array}\right| \\
& \rightarrow \frac{(-1)^{n}}{z^{q(n+1)}}\left|\begin{array}{ccccc}
1 & \left(\begin{array}{l}
0 \\
0
\end{array}\right) z^{q} & 0 & \cdots & 0 \\
x & \left(\begin{array}{l}
1 \\
0
\end{array}\right) m_{1}(z, y, q) & \left(\begin{array}{c}
1 \\
1
\end{array}\right) z^{q} & \cdots & 0 \\
x^{2} & \left(\begin{array}{l}
2 \\
0
\end{array}\right) m_{2}(z, y, q) & \left(\begin{array}{l}
2 \\
1
\end{array}\right) m_{1}(z, y, q) & \cdots & 0 \\
\ldots & \ldots & \ldots & \ddots & \ldots \\
x^{n-2} & \left(\begin{array}{c}
n-2 \\
0
\end{array}\right) m_{n-2}(z, y, q) & \left(\begin{array}{c}
n-2 \\
1
\end{array}\right) m_{n-3}(z, y, q) & \ldots & 0 \\
x^{n-1} & \left(\begin{array}{c}
n-1 \\
0
\end{array}\right) m_{n-1}(z, y, q) & \left(\begin{array}{c}
n-1 \\
1
\end{array}\right) m_{n-2}(z, y, q) & \ldots & \left(\begin{array}{c}
n-1 \\
n-1
\end{array}\right) z^{q} \\
x^{n} & \left(\begin{array}{c}
n \\
0
\end{array}\right) m_{n}(z, y, q) & \left(\begin{array}{c}
n \\
1
\end{array}\right) m_{n-1}(z, y, q) & \cdots & \left(\begin{array}{c}
n \\
n-1
\end{array}\right) m_{1}(z, y, q)
\end{array}\right|
\end{aligned}
$$

as $t$ tends to 0 . The determinantal expression (4.1) is thus proved.

Applying 3.3 to the determinant in 4.1 gives

$$
\begin{gathered}
(-1)^{n} z^{q(n+1)} F_{n}(x, y, z, q)=\left(\begin{array}{c}
n \\
n-1
\end{array}\right) m_{1}(z, y, q)(-1)^{n-1} z^{q n} F_{n-1}(x, y, z, q) \\
+(-1)^{n-1+1} x^{n} z^{q n}(-1)^{1-2} z^{q(1-1)} F_{1-2}(x, y, z, q) \\
+\sum_{r=2}^{n}(-1)^{n-r+1}\left(\begin{array}{c}
n \\
r-2
\end{array}\right) m_{n-r+2}(z, y, q) z^{q(n-r+1)}(-1)^{r-2} z^{q(r-1)} F_{r-2}(x, y, z, q),
\end{gathered}
$$

where $F_{-1}(x, y, z, q)=-1$. This can be simplified as 4.2 . The proof of Theorem 4.1 is complete.

\section{Relations Between Fubini and Eulerian polynomials}

It is known 23, 29] that Eulerian polynomials $A_{n}(t)$ for $n \geq 0$ can be generated by

$$
\frac{1-t}{e^{x(t-1)}-t}=\sum_{n=0}^{\infty} A_{n}(t) \frac{x^{n}}{n !}, \quad t \neq 1
$$

and that higher order Eulerian polynomials $A_{n}^{(\alpha)}(t)$ for $n \geq 0$ and $\alpha \in \mathbb{R}$ can be generated by

$$
\left[\frac{1-t}{e^{x(t-1)}-t}\right]^{\alpha}=\sum_{n=0}^{\infty} A_{n}^{(\alpha)}(t) \frac{x^{n}}{n !}, \quad t \neq 1 .
$$

Comparing (1.2) with 5.1) and (5.2) reveals that

$$
A_{n}(2)=A_{n}^{(1)}(2)=F_{n}=F_{n}(1)=F_{n}(0,1)=F_{n}^{(1)}(0,1)=y F_{n}(0, y, y, 1), \quad n \geq 0 .
$$


The equations (1.4) and (5.1) can be rewritten as

$$
\frac{1}{e^{w}-\left(1+\frac{1}{y}\right)}=-y \sum_{n=0}^{\infty} F_{n}(y) \frac{w^{n}}{n !}
$$

and

$$
\frac{1}{e^{w}-t}=-\sum_{n=0}^{\infty} \frac{A_{n}(t)}{(t-1)^{n+1}} \frac{w^{n}}{n !}
$$

This means that

$$
F_{n}(y)=y^{n} A_{n}\left(1+\frac{1}{y}\right)
$$

and

$$
A_{n}(t)=(t-1)^{n} F_{n}\left(\frac{1}{t-1}\right) .
$$

Similarly, the equations 1.5$)$ and $(5.2)$ can be rewritten as

$$
\frac{1}{\left[e^{w}-\left(1+\frac{z}{y}\right)\right]^{q}}=y^{q} e^{-x w} \sum_{n=0}^{\infty} F_{n}(x, y, z, q) \frac{w^{n}}{n !}
$$

and

$$
\frac{1}{\left(e^{w}-t\right)^{\alpha}}=\sum_{n=0}^{\infty} \frac{A_{n}^{(\alpha)}(t)}{(1-t)^{n+\alpha}} \frac{w^{n}}{n !} .
$$

Then we can conclude that

$$
F_{n}(0, y, z, q)=(-1)^{n+q} \frac{y^{n}}{z^{n+q}} A_{n}^{(q)}\left(1+\frac{z}{y}\right)
$$

and

$$
A_{n}^{(\alpha)}(t)=(1-t)^{n+\alpha} F_{n}(0,1, t-1, \alpha) .
$$

In 23. Theorem 1], it was obtained that Eulerian polynomials $A_{n}(t)$ and higher order Eulerian polynomials $A_{n}^{(\alpha)}(t)$ for $n \geq 0$ and $\alpha>0$ can be computed by

$$
A_{n}(t)=\sum_{k=0}^{n} k ! S(n, k)(t-1)^{n-k}
$$

and

$$
A_{n}^{(\alpha)}(t)=\frac{1}{\Gamma(\alpha)} \sum_{k=0}^{n} \Gamma(k+\alpha) S(n, k)(t-1)^{n-k},
$$

where $\Gamma(z)$ denotes the classical Euler gamma function [17, 25). Taking $t=2$ in (5.7) and using (5.3) recover (1.1). Substituting (5.5) into (5.7) yields

$$
(t-1)^{n} F_{n}\left(\frac{1}{t-1}\right)=\sum_{k=0}^{n} k ! S(n, k)(t-1)^{n-k}
$$

which is equivalent to (1.3). Combining (5.6) and (5.8) results in

$$
F_{n}(0,1, t-1, \alpha)=\frac{1}{\Gamma(\alpha)} \sum_{k=0}^{n}(-1)^{n-k} \frac{\Gamma(k+\alpha) S(n, k)}{(1-t)^{k+\alpha}} .
$$


Substituting (5.4) into (2.1), 3.1), and (3.2) arrives at a nice determinantal expression

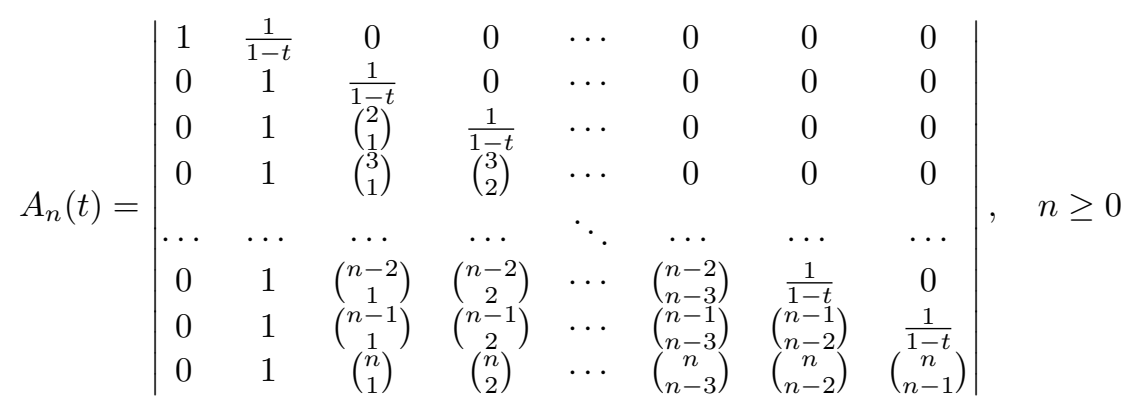

and two recurrence relations

$$
\begin{gathered}
A_{n}(t)=(t-1)^{n-1} \sum_{r=0}^{n-1}\left(\begin{array}{l}
n \\
r
\end{array}\right) \frac{A_{r}(t)}{(t-1)^{r}}, \quad n \in \mathbb{N}, \\
A_{n}(t)=(-1)^{n+1}(t-1)^{n-1} t\left[1-t+\sum_{k=0}^{n-1}(-1)^{k}\left(\begin{array}{l}
n \\
k
\end{array}\right) \frac{A_{k}(t)}{(t-1)^{k}}\right], \quad n \geq 0 .
\end{gathered}
$$

Theorem 2 in 23 , reads that the function

$$
F(t, x)=\frac{1}{e^{x(t-1)}-t}, \quad t \neq 1
$$

satisfies the nonlinear ordinary differential equation

$$
\frac{\partial^{n} F(t, x)}{\partial x^{n}}=(t-1)^{n} \sum_{i=0}^{n}\left[\sum_{k=i}^{n}(-1)^{k} k ! S(n, k)\left(\begin{array}{c}
k \\
i
\end{array}\right)\right] t^{i} F^{i+1}(t, x), \quad n \geq 0 .
$$

The equation (5.9) was simplified in [29, Theorem 5] as

$$
\frac{\partial^{n} F(t, x)}{\partial x^{n}}=(1-t)^{n} \sum_{k=0}^{n} S(n+1, k+1) k ! t^{k} F^{k+1}(t, x), \quad n \geq 0 .
$$

As an inversion formula of 5.10 , Theorem 4 in [29] states that

$$
F^{n+1}(t, x)=\frac{1}{n ! t^{n}} \sum_{i=0}^{n} \frac{s(n+1, i+1)}{(1-t)^{i}} \frac{\partial^{i} F(t, x)}{\partial x^{i}}, \quad n \geq 0,
$$

where $s(n, k)$, which can be generated by

$$
\frac{[\ln (1+x)]^{k}}{k !}=\sum_{n=k}^{\infty} s(n, k) \frac{x^{n}}{n !}, \quad|x|<1,
$$

stands for the Stirling numbers of the first kind. Taking $t=2$ in (5.10) and (5.11) leads to

$$
G^{(n)}(x)=(-1)^{n} \sum_{k=0}^{n}(-1)^{k} 2^{k} k ! S(n+1, k+1) G^{k+1}(x), \quad n \geq 0
$$

and

$$
G^{n+1}(x)=\frac{(-1)^{n}}{n ! 2^{n}} \sum_{i=0}^{n}(-1)^{i} s(n+1, i+1) G^{(i)}(x), \quad n \geq 0 .
$$


The equations (5.12) and (5.13) were also established in [19, Theorem 1] by three alternative approaches. By similar methods to those in [23, 19, 28, 29, 31] and closely related references therein, we can easily deduce

$$
\frac{\partial^{n} G(t, y)}{\partial t^{n}}=(-1)^{n} \sum_{m=0}^{n}(-1)^{m}(1+y)^{m} m ! S(n+1, m+1) G^{m+1}(t, y), \quad n \geq 0
$$

and

$$
G^{n+1}(t, y)=\frac{(-1)^{n}}{n !(1+y)^{n}} \sum_{m=0}^{n}(-1)^{m} s(n+1, m+1) \frac{\partial^{m} G(t, y)}{\partial t^{m}}, \quad n \geq 0 .
$$

In [23, Theorem 3] and [29, Theorem 6], it was obtained that

$$
\sum_{k=0}^{n}(-1)^{k}\left(\begin{array}{l}
n \\
k
\end{array}\right)\left[\frac{S(n-k, 0)}{(1-t)^{k-1}}+\frac{S(n-k, 1)}{(1-t)^{k}}\right] A_{k}(t)=0, \quad n \in \mathbb{N}
$$

and

$$
\sum_{k=0}^{n} \frac{s(n, k)}{(t-1)^{k}} A_{k}(t)=\frac{n !}{(t-1)^{n}}, \quad n \geq 0 .
$$

Substituting (5.5) into (5.14) and (5.15) arrives at

$$
\sum_{k=0}^{n}\left(\begin{array}{l}
n \\
k
\end{array}\right)\left[S(n-k, 1)-\frac{S(n-k, 0)}{t}\right] F_{k}(t)=0, \quad n \in \mathbb{N}
$$

and

$$
\sum_{k=0}^{n} s(n, k) F_{k}(t)=n ! t^{n}, \quad n \geq 0
$$

\section{REMARKS}

Finally, we list several remarks.

Remark 6.1. When $y=1$, the recurrence relations 3.1 and 3.2 becomes

$$
F_{n}=\sum_{r=0}^{n-1}\left(\begin{array}{l}
n \\
r
\end{array}\right) F_{r}, \quad n \in \mathbb{N}
$$

and

$$
F_{n}=(-1)^{n} 2\left[1-\sum_{k=0}^{n-1}(-1)^{k}\left(\begin{array}{l}
n \\
k
\end{array}\right) F_{k}\right], \quad n \geq 0 .
$$

These two recurrence relations appeared in [8] in different forms.

Remark 6.2. Let $u=u(t)=y\left(e^{t}-1\right)$. Then, by the Faà di Bruno formula 4.3 and the identities (4.4) and 4.5), we have

$$
\begin{aligned}
& \lim _{t \rightarrow 0} \frac{\mathrm{d}^{n}}{\mathrm{~d} t^{n}} \frac{1}{1-y\left(e^{t}-1\right)}=\lim _{t \rightarrow 0} \sum_{k=0}^{n} \frac{\mathrm{d}^{k}}{\mathrm{~d} u^{k}} \frac{1}{1-u} \mathrm{~B}_{n, k}\left(y e^{t}, y e^{t}, \ldots, y e^{t}\right) \\
= & \lim _{t \rightarrow 0} \sum_{k=0}^{n} \frac{k !}{(1-u)^{k+1}}\left(y e^{t}\right)^{k} \mathrm{~B}_{n, k}(1,1, \ldots, 1)=\sum_{k=0}^{n} S(n, k) k ! y^{k}=F_{n}(y) .
\end{aligned}
$$


This recovers once again that the Fubini numbers and polynomials $F_{n}$ and $F_{n}(y)$ have generating functions $\frac{1}{2-e^{t}}$ and $\frac{1}{1-y\left(e^{t}-1\right)}$.

Remark 6.3. On 24 July 2018, when commenting on the paper [29] on ResearchGate, Professor Dr. Boyadzhiev (Ohio Northern University) recommended the website http://www.luschny.de/ math/euler/EulerianPolynomials.html and two papers [3, 9. He said that, when writing about Eulerian polynomials, it is good to mention that they originate from Euler and their theory is very classical - generating function and other formulas have been known for very long time. He pointed out that the paper 3 is a review where Eulerian polynomials are linked to geometric polynomials and Apostol-Bernoulli polynomials.

On 15 May 2019, Professor Dr. Boyadzhiev (Ohio Northern University) commented on ResearchGate again that the author should take in consideration the paper [4] and that the name "geometric polynomials" is established in the mathematics community while "Fubini polynomials" is not.

Remark 6.4. The ideas in this paper and the formulas 2.2 and 3.3 have been utilized in the papers $20,22,24,26,27,30,32,33,34,35$, and closely related references therein.

Remark 6.5. This paper is an extension of the preprint [18] and a slight revision of the preprint [15].

\section{REFERENCES}

[1] N. Bourbaki, Elements of Mathematics: Functions of a Real Variable: Elementary Theory, Translated from the 1976 French original by Philip Spain. Elements of Mathematics (Berlin). Springer-Verlag, Berlin, 2004; Available online at http://dx.doi.org/10.1007/978-3-642-59315-4

[2] K. N. Boyadzhiev, A series transformation formula and related polynomials, Int. J. Math. Math. Sci. 2005, no. 23, 3849-3866; Available online at https://doi.org/10.1155/IJMMS.2005.3849

[3] K. N. Boyadzhiev, Apostol-Bernoulli functions, derivative polynomials and Eulerian polynomials, Adv. Appl. Discrete Math. 1 (2008), no. 2, 109-122.

[4] K. N. Boyadzhiev and A. Dil, Geometric polynomials: properties and applications to series with zeta values, Anal. Math. 42 (2016), no. 3, 203-224; Available online at https://doi.org/10.1007/s10476-016-0302-y

[5] N. D. Cahill, J. R. D'Errico, D. A. Narayan, and J. Y. Narayan, Fibonacci determinants, College Math. J. 33 (2002), no. 3, 221-225; Available online at https://doi.org/10.2307/1559033

[6] L. Comtet, Advanced Combinatorics: The Art of Finite and Infinite Expansions, Revised and Enlarged Edition, D. Reidel Publishing Co., Dordrecht and Boston, 1974; Available online at https://doi.org/10.1007/ 978-94-010-2196-8.

[7] M. E. Dasef and S. M. Kautz, Some sums of some importance, College Math. J. 28 (1997), 52-55.

[8] A. Dil and V. Kurt, Investigating geometric and exponential polynomials with Euler-Seidel matrices, J. Integer Seq. 14 (2011), no. 4, Article 11.4.6, 12 pp.

[9] D. Foata, Eulerian polynomials: from Euler's time to the present, In: The Legacy of Alladi Ramakrishnan in the Mathematical Sciences, 253-273, Springer, New York, 2010; Avaialble online at https://doi.org/10.1007/ 978-1-4419-6263-8_15.

[10] O. A. Gross, Preferential arrangements, Amer. Math. Monthly 69 (1962), 4-8; Available online at https: //doi.org/10.2307/2312725

[11] R. D. James, The factors of a square-free integer, Canad. Math. Bull. 11 (1968), 733-735; Available online at https://doi.org/10.4153/CMB-1968-089-7.

[12] L. Kargin, Some formulae for products of Fubini polynomials with applications, arXiv preprint (2016), available online at https://arxiv.org/abs/1701.01023

[13] D. S. Kim, T. Kim, H.-I. Kwon, and J.-W. Park, Two variable higher-order Fubini polynomials, J. Korean Math. Soc. 55 (2018), no. 4, 975-986; Available online at https://doi.org/10.4134/JKMS.j170573

[14] F. Qi, Derivatives of tangent function and tangent numbers, Appl. Math. Comput. 268 (2015), 844-858; Available online at http://dx.doi.org/10.1016/j.amc.2015.06.123

[15] F. Qi, Determinantal expressions and recurrence relations for Fubini and Eulerian polynomials, HAL preprint (2018), available online at https://hal.archives-ouvertes.fr/hal-01853686v2 
[16] F. Qi, Diagonal recurrence relations, inequalities, and monotonicity related to the Stirling numbers of the second kind, Math. Inequal. Appl. 19 (2016), no. 1, 313-323; Available online at https://doi.org/10.7153/mia-19-23

[17] F. Qi, Limit formulas for ratios between derivatives of the gamma and digamma functions at their singularities, Filomat 27 (2013), no. 4, 601-604; Available online at http://dx.doi.org/10.2298/FIL1304601Q.

[18] F. Qi, On generalized Fubini polynomials, HAL preprint (2018), available online at https://hal. archives-ouvertes.fr/hal-01853686v1

[19] F. Qi, Simplification of coefficients in two families of nonlinear ordinary differential equations, Turkish J. Anal. Number Theory 6 (2018), no. 4, 116-119; Available online at https://doi.org/10.12691/tjant-6-4-2

[20] F. Qi, V. Čerňanová, and Y. S. Semenov, Some tridiagonal determinants related to central Delannoy numbers, the Chebyshev polynomials, and the Fibonacci polynomials, Politehn. Univ. Bucharest Sci. Bull. Ser. A Appl. Math. Phys. 81 (2019), no. 1, 123-136.

[21] F. Qi and R. J. Chapman, Two closed forms for the Bernoulli polynomials, J. Number Theory 159 (2016), 89-100; Available online at http://dx.doi.org/10.1016/j.jnt.2015.07.021

[22] F. Qi and B.-N. Guo, A determinantal expression and a recurrence relation for the Euler polynomials, Adv. Appl. Math. Sci. 16 (2017), no. 9, 297-309.

[23] F. Qi and B.-N. Guo, Explicit formulas and recurrence relations for higher order Eulerian polynomials, Indag. Math. 28 (2017), no. 4, 884-891; Available online at https://doi.org/10.1016/j.indag.2017.06.010.

[24] F. Qi and B.-N. Guo, Expressing the generalized Fibonacci polynomials in terms of a tridiagonal determinant, Matematiche (Catania) 72 (2017), no. 1, 167-175; Available online at https://doi.org/10.4418/2017.72.1.13

[25] F. Qi and B.-N. Guo, Integral representations and complete monotonicity of remainders of the Binet and Stirling formulas for the gamma function, Rev. R. Acad. Cienc. Exactas Fís. Nat. Ser. A Math. RACSAM 111 (2017), no. 2, 425-434; Available online at https://doi.org/10.1007/s13398-016-0302-6.

[26] F. Qi and B.-N. Guo, Some determinantal expressions and recurrence relations of the Bernoulli polynomials, Mathematics 4 (2016), no. 4, Article 65, 11 pages; Available online at https://doi.org/10.3390/math4040065

[27] F. Qi and B.-N. Guo, Two nice determinantal expressions and a recurrence relation for the Apostol-Bernoulli polynomials, J. Indones. Math. Soc. (MIHMI) 23 (2017), no. 1, 81-87; Available online at https://doi.org/ 10.22342/jims.23.1.274.81-87

[28] F. Qi, D. Lim, and B.-N. Guo, Explicit formulas and identities for the Bell polynomials and a sequence of polynomials applied to differential equations, Rev. R. Acad. Cienc. Exactas Fís. Nat. Ser. A Mat. RACSAM 113 (2019), no. 1, 1-9; Available online at https://doi.org/10.1007/s13398-017-0427-2

[29] F. Qi, D. Lim, and B.-N. Guo, Some identities related to Eulerian polynomials and involving the Stirling numbers, Appl. Anal. Discrete Math. 12 (2018), no. 2, 467-480; Available online at https://doi.org/10.2298/ AADM171008014Q

[30] F. Qi and A.-Q. Liu, Alternative proofs of some formulas for two tridiagonal determinants, Acta Univ. Sapientiae Math. 10 (2018), no. 2, 287-297; Available online at https://doi.org/10.2478/ausm-2018-0022

[31] F. Qi, D.-W. Niu, and B.-N. Guo, Some identities for a sequence of unnamed polynomials connected with the Bell polynomials, Rev. R. Acad. Cienc. Exactas Fís. Nat. Ser. A Math. RACSAM 113 (2019), no. 2, 557-567; Available online at https://doi.org/10.1007/s13398-018-0494-z

[32] F. Qi, J.-L. Wang, and B.-N. Guo, A determinantal expression for the Fibonacci polynomials in terms of a tridiagonal determinant, Bull. Iranian Math. Soc. 45 (2019), no. 6, 1821-1829; Available online at https: //doi.org/10.1007/s41980-019-00232-4

[33] F. Qi, J.-L. Wang, and B.-N. Guo, A representation for derangement numbers in terms of a tridiagonal determinant, Kragujevac J. Math. 42 (2018), no. 1, 7-14; Available online at https://doi.org/10.5937/ KgJMath1801007F

[34] F. Qi and J.-L. Zhao, Some properties of the Bernoulli numbers of the second kind and their generating function, Bull. Korean Math. Soc. 55 (2018), no. 6, 1909-1920; Available online at https://doi.org/10.4134/BKMS. b180039.

[35] F. Qi, J.-L. Zhao, and B.-N. Guo, Closed forms for derangement numbers in terms of the Hessenberg determinants, Rev. R. Acad. Cienc. Exactas Fís. Nat. Ser. A Mat. RACSAM 112 (2018), no. 4, 933-944; Available online at https://doi.org/10.1007/s13398-017-0401-z

[36] S. M. Tanny, On some numbers related to the Bell numbers, Canad. Math. Bull. 17 (1974/75), no. 5, 733-738; Available online at https://doi.org/10.4153/CMB-1974-132-8 